\title{
Evaluating the Utility of Tablet PCs in a Software Engineering Capstone Course
}

\author{
Jiajia Zhang \\ Dept. of Electrical \& \\ Computer Engineering \\ University of Alberta \\ jiajia3@ece.ualberta.ca
}

\author{
Scott Dick \\ Dept. of Electrical \& \\ Computer Engineering \\ University of Alberta \\ dick@ece.ualberta.ca
}

\author{
Nelson Durdle \\ Dept. of Electrical \& \\ Computer Engineering \\ University of Alberta \\ durdle@ece.ualberta.ca
}

\begin{abstract}
The ECE Dept. at the University of Alberta uses Tablet PCs as a key resource in the Software Engineering capstone design course. We are currently developing a survey instrument (piloted in 2009) to measure the utility of these Tablets. The instrument is based on technology adoption theory, which posits that specific psychological states are antecedents to the usage of a new technology. We perform an exploratory factor analysis of the pilot survey, with an emphasis on our operationalization of the construct of Use of the Technology. However, small-sample-size effects prevent us from fully validating the model.
\end{abstract}

\section{Introduction}

Educational technology has become both an important part of the post-secondary educational landscape, and a major budget priority. As one example, the Knowledge Infrastructure Program from the Canadian federal government earmarks $\$ 600$ million for technology upgrades at colleges and universities across the nation. This is intended as a $50 \%$ matching fund, and so a total of $\$ 1.2$ billion is expected to be committed over the two-year life of the program [4]. With such large dollar amounts at stake, the potential for waste is obvious; thus, evidence-based guidelines on effective technological supports for education are clearly needed. However, information technology is in perpetual flux, and thus the matching between a particular technology and pedagogical objective (i.e. task-technology fit [10]) will change over time This implies an ongoing need to evaluate the impact and utility of new and changing technologies for students in a variety of pedagogical contexts.

In 2008 the ECE Department at the University of Alberta received a donation of Tablet PCs as part of a Hewlett-Packard Technology for Teaching (TfT) Grant, for use in the Computer Engineering (Software Option) capstone design course (CMPE 440). We use these Tablet PCs as the development environment for our BlackBerry-based mobile computing laboratory environment. A Tablet PC is issued to each student in CMPE 440 for the duration of the semester, and is pre- loaded with a complete BlackBerry integrated development environment. In order to evaluate the utility of the Tablet PCs, we piloted a survey instrument at the end of the Winter 2009 semester. The survey is based on the well-known Technology Adoption Model (TAM) [7], which posits that the constructs of Attitude, Usefulness and Ease of Use influence the dependent variable Use. Scales for Attitude (5-point semantic differential scale), Usefuleness, and Ease of Use (both 7-point Likert scales) are widely available in the literature. The construct of Use is a somewhat different case; many studies of technology adoption operationalize it via the construct "Intention to Use," owing to the difficulty of measuring actual use. Other studies, however, have developed instruments to measure actual usage [1, 13].

Our goal in this article is to propose a multi-item scale for measuring the construct of Use, and validating it through an exploratory factor analysis. This scale was part of a survey instrument administered to the students in CMPE 440 during the Winter 2009 term. We found that, in the main, our proposed scale for Use indeed appears to be unidimensional; however, two out of nine items should be dropped (one of them because it duplicates responses for another item).

The remainder of this paper is organized as follows. Essential background on Tablet PCs and technology acceptance theory is reviewed in Section 2. The educational context of the survey is described in Section 3, and our survey and data analysis is discussed in Section 5. Conclusions and a discussion of future work are offered in Section 5.

\section{Review of Related Work}

\subsection{Tablet PCs in Education}

Up until 2010, the Tablet PC has essentially been a niche product. The most familiar is the "convertible" style, which traditionally has a full QWERTY keyboard with a pointing device (pointing stick or touchpad), and a rotatable screen. When rotated, the screen usually locks in position over the keyboard, 
providing a slate-like writing surface. The signature input modality of these devices is the stylus, which allows the user to write or draw on the touch-sensitive screen. A different variety of Tablet, sometimes called a "slate," does away with the QWERTY keyboard, and only uses a touch-based interface. Typically, Tablets are deployed in environments where the convenience of a slate-type interface is high (health care, field \& construction work, etc); it would be fair to say that these applications do not include entry of voluminous text [3].

In 2010, Apple has introduced the iPad device, which is a variation on the slate-style computer. The iPad does not have a stylus, but instead relies on fingertip gestures for user input. By providing an onscreen touch keyboard (similar to the iPhone), users obtain many of the advantages of the convertible-style Tablet, without the physical weak point of the rotating hinge. Current market forecasts indicate that tens of millons of Tablet devices could be sold this year, with iPad sales expected to be prominent among them [18]. As with all mobile devices, however, there are tradeoffs; it remains to be seen if the stylus (with its associated "digital ink" capability) is an input modality that users still find useful. We do suspect that a stylus will remain important to a niche of users that need fairly precise free-form data entry and drawing. The fingertip is currently an inherently imprecise pointing device; in the context of our work, software design diagrams are detailed documents containing text and free-form drawing; a fingertip interface for creating such documents does not currently seem feasible.

There is a modest but growing literature on the use of TPCs in education, mostly focused on postsecondary classes [11]. While some of this literature focuses on faculty use of TPCs, we will confine our review to the literature on student TPC use. A large portion or the literature discusses TPCs equipped with a limited software inventory; DyKnow , Microsoft OneNote and Office, and Classroom Presenter are frequently discussed. From this literature, two messages seem to clearly stand out: first, the question of task-technology fit (see below) is absolutely essential. When this fit appears strong (e.g. [16]) clear evidence of improved learning outcomes is commonly reported. When the task-technology fit appears weaker, learning outcomes are essentially unchanged (e.g. [5, 12]). The second lesson is that, while the TPC's new affordances offer great opportunities, we cannot forget its limitations. One example is the choice of the more familiar keyboard over the (often slower) pen for text input. Training in TPC use is also commonly needed, as the software is usually new to students [11]. The TPC also shares many of the ergonomic problems of laptops while adding some new ones; pen-based input is convenient in a number of awkward positions, but this leads to musculoskeletal discomfort quite rapidly [17].

\subsection{Technology Acceptance Theory}

For obvious reasons, there is great interest in understanding why some technological innovations flourish, while others fall by the wayside. Technology Acceptance Theory is the branch of Information Science that seeks to answer this question. This area draws heavily on psychological theory, and develops additional models to try and explain the complex decision to use or not use a given innovation. One of the most influential ideas is the Theory of Reasoned Action (TRA) [2] from psychology, which posits that a person's beliefs influence their attitudes towards a situation (a decision to make, an action to take), which in turn influence the person's intentions. Intentions are also shaped by subjective norms, which are that individual's perceptions of how people whose opinions they respect would expect them to behave. Intentions then lead to observable actions (the rest of course being unobservable psychological states). It is the foundation for several other theoretical models:

- The Theory of Planned Behavior (TPB) adds the construct of "behavioural control," defined as perceived constraints (intrinsic or extrinsic) on the individual's behaviour [20]

- The Technology Acceptance Model (TAM) specializes TRA for the case of deciding whether or not to use a technology. Two beliefs are identified: Perceived Usefulness and Perceived Ease of Use, which are posited to influence Attitude towards a technology. Those attitudes influence the Intention to Use that technology, which finally influences its Use [7]. Subjective norms from TRA were added as another factor in the TAM2 refinement [22]. TAM is one of the best-known models in this area.

- The C-TAM-TBP model incorporates factors from both TAM and TBP.

Social Cognitive Theory (SCT) also has a prominent role. In general, SCT is a theory of how people learn new skills and behaviors in a social setting. It explicitly incorporates the person's "inner life," and how cognition, emotion and reflection shape our behaviours and reactions to our environment - and how the environment and the outcomes of our behaviours alter our thoughts and beliefs ("reciprocal determinism"). SCT was specialized for computer use in [6], by narrowing the construct of self-efficacy to focus on self-efficacy in computer use. A scale for measuring this construct was developed and validated. 
More generally, the construct self-efficacy in using "innovation $Y$ " allows us to employ SCT in the general technology-acceptance field.

Motivation theory is also used to study technology acceptance. Generally, motivations can be categorized as intrinsic or extrinsic (arising from within or without of the person). In the technology acceptance context, intrinsic motivations involve no apparent reinforcement for behaviour other than the behaviour itself; while extrinsic motivations are an individual's assessment of the instrumentality of the behaviour in achieving their goals [8].

More specific models focusing just on technology acceptance have also been developed. Rogers [15] proposes five factors to account for the uptake of a new technology: the advantages of the innovation over existing technologies (relative advantage); how consistent the innovation is with the values, needs and experiences of users (compatibility); how easily users can comprehend and use the technology on an ongoing basis (complexity); how easily users can set up and experiment with this innovation for the first time (trialability); and how visible the benefits of using the innovation are to users (observability). The Model of PC Utilization (MPCU) is a specialization of Triandis' theory of human behaviour for predicting PC utilization; it has subsequently been used in more general studies of technology acceptance. This theory posits 6 perceptions that influence use of an innovation: job-fit (instrumentality of the innovation in performing job functions), complexity (as in Rogers), long-term consequences (whether adopting the innovation will provide long-term benefit), affect towards use (emotional reactions to using the innovation), social factors (subjective perceptions of cultural norms and interpersonal agreements affecting use of the innovation), and facilitating conditions (objective conditions that make using the innovation easier) [21]. Venkatesh et al. have proposed a model that employs concepts from these various theories (UTAUT), and provided evidence that it has considerably greater explanatory power that any one of the others alone [23].

One important element of technology acceptance is how effectively an innovation supports a user in their tasks. This is implicit in the "Usefulness" construct of TAM, and explicitly treated as the factor "Job-fit" in MPCU. However, in an influential article, Goodhue \& Thompson [10] argue that this concept (which they dub task-technology fit) is actually a multidimensional construct that goes beyond usage models like TAM or UTAUT. Fundamentally, the argument is that the instrumentality of an innovation cannot be captured in a uni-dimensional scale. Operationalization of the task-technology fit construct (as in $[9,10]$ ) is usually domain-specific, as tasks and technologies vary so widely. Generally speaking, tasktechnology fit decomposes into factors covering the correspondence between specific task requirements and technological functionality; the quality of the technology (reliability, timeliness, etc.); and the quality of technical support provided $[9,10]$.

\section{Educational Context}

\subsection{The Software Engineering Capstone Course}

The Computer Engineering program at the University of Alberta offers three degree streams: traditional computer engineering, a Software stream, and a Nanoscale System Design option. In the Software option, the degree emphasizes the construction of large-scale software systems. Students still take classical computer engineering courses including signal \& systems and digital logic, but will also have a heavier component of computing science courses (e.g. operating systems, databases) and specialized software engineering courses that cover core topics in the Software Engineering Body of Knowledge (SWEBOK). These include classes in requirements engineering, software design, construction, testing and maintenance, software quality, and project management. The program of study covers four years (five if students pursue co-op study), and by the final semester of year 4 they will have acquired the skills necessary to develop reliable, large scale software systems. At this point they will have their capstone design experience in the course CMPE 440.

As one would expect, CMPE 440 is not primarily a lecture class. The goal of the class if for students to work in groups to conceive, design and deliver a reasonably complex, working software system. The students are randomly assigned to project groups, but then have free choice of their project topic. Suggestions (some from local companies) are provided, or the students may suggest their own. They then write their own requirements document (subject to the Instructor's approval), and are accountable for it with $30 \%$ of the final grade dependent on meeting all functional requirements. Clearly, this is almost a pure constructionist pedagogy; this is, we feel, appropriate in a capstone course where all class members are "advanced" learners. The goal of the class is thus to challenge students to demonstrate what they have learned in the program; along the way they will certainly encounter the communication difficulties and frustrating software bugs that make software so notoriously difficult to build; this will be their first project that is large enough to surface these issues. We support them by monitoring progress on a weekly basis, providing collaborative-work tools, and having 
interim deliverables (requirements specifications, user interface prototypes) that mitigate procrastination. A one-hour weekly lecture serves as a regular point of contact, during which mobile application development is discussed.

\subsection{Technological Environment}

CMPE 440 is organized around a mobile computing environment in the ECE Dept.'s Software Engineering Laboratory, allowing students a wide choice of target environments for their capstone projects. The basic software engineering components of the laboratory include Linux-based desktops, together with a typical suite of development tools for Java (our principal development language), including current versions of Eclipse and the JUnit framework, network-based MySQL and CVS instances, and their own network directory space. In addition, in 2008 we acquired a set of BlackBerry 9000 smartphones (known as the Bold) as a target environment for J2ME-based mobile applications. The Bold boasts a half-VGA (480x320) display, full QWERTY keyboard, WiFi and Bluetooth radios, and a GPS receiver. It is thus capable of running complex and rich mobile applications.

The HP TfT grant complemented this environment, allowing us to significantly extend the range of target environments and configurations. Firstly, the Tablet PCs themselves are mobile devices, with a unique input modality - the pen-based "digital inking" capability. At the same time, they offer far greater memory, disk storage, and processing power (a dual-core $1.2 \mathrm{GHz}$ processor) than the smartphones. They furthermore have built-in Bluetooth and WiFi radios, offering the opportunity for wireless communication with the smartphones. HP's grant also included a WiFi access point, which we have used to create a private WiFi LAN in the laboratory. Student projects can thus target a traditional desktop environment; a Tablet PC environment; a BlackBerry smartphone; or a cross-platform system deployed on some or all of these environments. A good example in the 2010 year was a fitness-monitoring application that used the GPS receiver on the smartphone to track progress on an exercise route (for walking, cycling, etc.); a desktop component based on a datacube architecture gave users a sophisticated analysis of their progress towards their fitness goals.

In setting up the BlackBerry development environment (an Eclipse plugin), we also recognized that the Tablet PCs could be an element of electronic design notebooks for the class. Software engineering makes use of a number of collaborative development tools, including Wikis and shared code repositories. We decided that the requirements specification and user-interfaces prototypes would be posted on a shared Wiki; to ensure that the students all have equal access to this resource, we issue a Tablet PC to each student for the whole semester. We also determined that the Tablets would have the BlackBerry development environment loaded as part of their disk image; the Eclipse plugin only runs on Windows Vista or Windows 7, and so is not available on the Linux desktops. Thus, the Tablet PCs are an integral part of the CMPE 440 laboratory exercises (which introduce BlackBerry application development) and many of the capstone projects (in the 2010 year, 60\% of the projects had a significant BlackBerry component).

\section{Evaluating the Tablet PCs}

Our evaluation goal was to determine the utility of the Tablet PCs (and separately the course Wiki), which we operationalize as the decision to use the Tablets. Use of the Tablets is not mandatory, and the students are fully capable of and skilled in setting up their own development environments (in fact, evidence suggests that "Millennial" generation students prefer to co-opt and personalize IT resources on their own terms rather than being given a "solution" [14]). Thus, acceptance of the Tablet will clearly indicate the student's perception of its utility. This argument indicates that technology acceptance theory is an appropriate conceptual framework for the study. We choose to employ the TAM model; it is widely used, scales for each item (save Use) are readily available, but it is still relatively simple. Due to the reduced IT degree enrolments of three years ago, our survey population is only 11 students, and so we feel a parsimonious model is essential. The survey items are adapted from [7]; this gives us a 7-level semantic-differential scale for Attitude, and 7-point Likert scales (anchored on "Strongly Disagree and "Strongly Agree") for Usefulness and Ease of Use. There were 9 items that measured Use in our survey. 8 of these asked the students to report how often they used the Tablet to perform common tasks: checking email, working on the 440 project, any other coursework, accessing the Internet for coursework, accessing $U$ of Alberta Internet resources, accessing the Internet for personal use, engaging in collaborative work, and using the Tablet's Microsoft Office software. This is similar to the use pattern survey in [13] or the single "Use" item in [1]. The ninth question asked how much of a premium the student would be willing to pay to buy a Tablet PC over a standard laptop, and is similar to the survey item used in [19]. Responses were in an ordered 5-level multiple-choice format, with order-ofmagnitude differences between consecutive levels; the exception was WillingToPay, which was a 7-item scale with linear increases between consecutive levels. 
The surveys were administered during the final lecture in CMPE 440, Winter 2009. As students in a course are a particularly vulnerable population, $U$ of Alberta procedures require that the instructor not be present; surveys \& consents are administered, collected and held by a neutral $3^{\text {rd }}$-party faculty member, and only released once course grades are posted. The response rate was excellent with all 11 students electing to complete the survey, and all students completing all survey items.

In analyzing the survey responses we realized that the responses for "email use" and "accessing $U$ of Alberta Internet resources" were identical for all survey respondents. As this prevents the correlation matrix from being positive definite, we will exclude the UA Internet resources item from further analysis. We also determined that the WillingToPay item was poorly correlated with the remaining items (in fact, most respondents indicated that they were unwilling to pay any premium for a Tablet). The corrected ItemTotal correlation for WillingToPay was only 0.376, and our factor analysis also indicated that it should be removed. Given the large swings that the laptop market has experienced in the last few year (the rise of inexpensive Netbooks, the launch of iPad), price premium questions might also be unreliable due to rapidly-changing consumer expectations. Hence, we will only present the factor analysis results for the remaining seven items, excluding the WillingToPay and UA Internet items.

Our first question was whether the relatively small population was in fact sufficient to proceed with any analysis. We examine the Kaiser-Meyer-Olkin (KMO) sampling-adequacy statistic and Bartlett's test of sphericity for this purpose. The KMO statistic is 0.662, indicating a reasonable degree of common variance among the responses. Barlett's test rejects the null hypothesis of an identity correlation matrix with $\mathrm{p}$-value $<0.001$, indicating the presence of important correlations between items. Thus, proceeding with a factor analysis seems reasonable; however, we will be conservative in forming our conclusions due to the small sample size.

We next conduct an exploratory factor analysis using maximum-likelihood extraction. We find that the first eigenvector covers over $80 \%$ of the total variance in the dataset; a scree test (see Figure 1) indicates that we should only select this first factor for dimensionality reduction. Examining the factor loadings (see Table 1), we find that all variables load heavily on this component (between 0.71 and 0.99 for all variables). A goodness-of-fit test indicates that this model is appropriate $(\chi=45.673, d f=14, p<0.000)$.

The reliability of the scale is very good; Cronbach's Alpha for these seven items is 0.959. In fact, the reliability when considering all nine Use items is still high (Alpha=0.955).

Given these lines of evidence, we feel that our scale for Use should be adjusted by dropping the $U$ of A Internet Resources item (this appears to be synonymous is email use in the students minds) and the WillingToPay item (this does not seem to be correlated with the other items, and its face validity now seems questionable, as discussed). The resulting 7-item scale appears to be a psychometrically valid uni-dimensional scale at this stage of our research.

Scree Plot

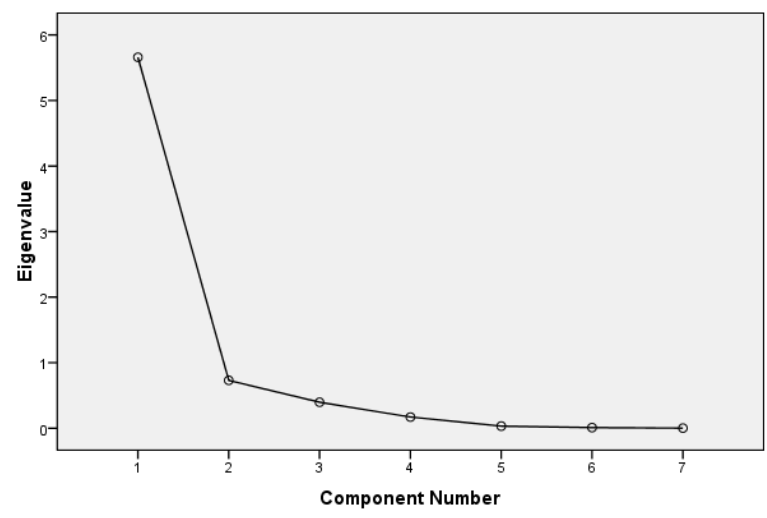

Figure 1: Scree Plot for “Use” Items

Table 1: Factor Loadings for "Use" Items

\begin{tabular}{|c|c|}
\hline Item & Factor 1 Loading \\
\hline OtherCoursework & 0.990 \\
\hline CollaborativeWork & 0.951 \\
\hline PersonalUse & 0.898 \\
\hline UseForCMPE440 & 0.870 \\
\hline UseForEmail & 0.865 \\
\hline InternetForCoursework & 0.854 \\
\hline UseMS-OfficeSuite & 0.717 \\
\hline
\end{tabular}

\section{Conclusions and Future Work}

We have presented a factor analysis of our operationalization of "Use" in evaluating the use of Tablet PCs in a software engineering capstone design course. Of the original nine items for measuring "Use," one was removed for being a duplicate of another item, while a second did not appear to correlate well with the remaining items. In addition, events in the IT product space call into question the face validity of that second item. At this time, our pool of responses is too small to validate the overall TAM model; specifically, the KMO sampling-adequacy statistic for Ease of Use is too low (0.262) to proceed with factor analysis. We believe that this is a problem of sample size; the KMO statistics for the remaining 
constructs of Attitude and Usefulness are adequate ( 0.748 and 0.737 , respectively). As the TAM model is widely used, we thus believe that this survey, if administered to an adequate sample of students, will provide useful results.

In future work, we will first repeat this survey for a larger sample of students. Enrolments for upcoming cohorts in the Computer Engineering program indicate that Winter 2009 was likely the nadir of enrolments in CMPE 440, meaning it is likely we will have a larger sample in coming years. In addition, the CMPE 440 lectures, and labs are in a steady state; and the demographics of upcoming cohorts appear relatively homogenous with the 2009 cohort. We will thus investigate the possibility of pooling results across cohorts to increase the power of our evaluation.

\section{References}

[1] R. Agarwal, E. Karahanna, "On the multidimensional nature of compatibility beliefs in technology acceptance," in Proc. DIGIT, Helsinki, Finland.

[2] I. Ajzen, M. Fishbein, Understanding attitudes and predicting social behavior. Englewood Cliffs, NJ, USA: Prentice-Hall, 1980.

[3] J. Beaudoin, "Survey finds physicians split over tablet PC," in HealthcareIT News. vol. Jan. 24 Menlo Park, CA, USA 2005.

[4] L. Bentacur, M. Mackenzie, "Canada: Educational Supplies and Technology Market," U.S. Commercial Service, USA Department of Commerce, Washington, D.C., USA 2009.

[5] A. Chidanandan, R. DeVasher, P. Ferro, D. Fisher, S. Mitra-Kirtley, L. D. Merkle, D. Mutchler, M. Simoni, S. Sexton, A. WattJ. M. Williams, "Evaluating the symbiosis of DyKnow software and pen-based computing in the Rose-Hulman classroom," in Proceedings of WIPTE, J. Prey, et al., Eds.: Purdue Univ. Press, 2007, pp. 21-31.

[6] D. R. Compeau, C. A. Higgins, "Computer selfefficacy: development of a measure and test," MIS Quarterly, vol. 19, pp. 189-211, 1995.

[7] F. D. Davis, "User accptance of information systems: The technology acceptance model (TAM)," Graduate School of Business, University of Michigan, 1987.

[8] F. D. Davis, R. P. Bagozzi, P. R. Warshaw, "Extrinsic and intrinsic motivation to use computers in the workplace," J. Applied Social Psychology vol. 22, pp. 1111-1132, 1992.

[9] M. T. Dishaw, D. M. Strong, "Extending the technology acceptance model with tasktechnology fit constructs," Inf \& Mgmnt, vol. 36, pp. 9-21, 1999.

[10] D. L. Goodhue, R. L. Thompson, "Tasktechnology fit and individual performance," MIS Quarterly, vol. 19, pp. 213-236, 1995.
[11] G. Neal, K. Davisdson, "Contesting ideas of innovative teaching practice with tablet PCs," in Proc. AARE 2008, 2008.

[12] H. T. Nguyen, S. G. Bilen, R. DevonJ. Wise, "Adopting tablet PCs in design education: student use of tablet PCs and lessons learned," in Proc. World Conf. E-Learning in Corp., Govern., Health, and Higher Ed., 2007, pp. 1172-1177.

[13] F. Prescod, L. Dong, "Learning Style Trends and Laptop Use Patterns:Implication for Students in an IT Business School," in Proc. ISECON, Dallas, TX, USA, 2006, p. 6 pp.

[14] P. Resta, T. Laferriere, "Technology in support of collaborative learning," Educ Psychol Rev vol. 19, pp. 65-83, 2007.

[15] E. Rogers, Diffusion of Innovations, 4th Ed. New York, NY: Free Press, 1995.

[16] D. G. Schulze, R. R. Struthers, P. R. OwensG. E. Van Scoyoc, "Teaching soil-landscape interactions using rugged tablet PCs in the field," in Proceedings of WIPTE, J. Prey, et al., Eds.: Purdue Univ. Press, 2007, pp. 119-126.

[17] C. M. Sommerich, K. Collura, "Learning with mobile technology in high school: a human factors perspective," in Proceedings of WIPTE J. Prey, et al., Eds.: Purdue Univ. Press, 2007, pp. 127-136.

[18] Staff, "Tablet computer market to boom: Deloitte," in AFP. vol. Jan. 19 San Francisco, 2010.

[19] B. Surgenor, K. Firth, "Tablet PC Technology for an Enhanced Engineering Capstone Design Course," in Hewlett-Packard Technology for Teaching Worldwide Conference, San Diego, CA, USA, 2007.

[20] S. Taylor, P. A. Todd, "Understanding information technology usage: a test of competing models," Information Systems Research, vol. 6, pp. 144176, 1995.

[21] R. L. Thompson, C. A. Higgins, J. M. Howell, "Personal computing: towards a conceptual model of utilization," MIS Quarterly, vol. 15, pp. 124143, 1991.

[22] V. Venkatesh, F. D. Davis, "A theoretical extension of the technology acceptance model:four longitudinal field studies," Management Science, vol. 45, pp. 186-204, 2000.

[23] V. Venkatesh, M. G. Morris, G. B. DavisF. D. Davis, "User acceptance of information technology: toward a unified view," MIS Quarterly, vol. 27, pp. 425-478, 2003. 